\title{
Contrast-Enhanced 3T High-Resolution MR Imaging in Symptomatic Atherosclerotic Basilar Artery Stenosis
}

\author{
X. Lou, N. Ma, L. Ma, and W.-J. Jiang
}

\section{ABSTRACT}

BACKGROUND AND PURPOSE: Contrast-enhanced 3T high-resolution MR imaging can be used to determine the wall enhancement pattern of the basilar artery in symptomatic atherosclerotic stenosis. We used this method to explore the relationship between wall enhancement and both recent infarction in the territory of the stenotic BA and subsequent ischemic events associated with the stenotic BA.

MATERIALS AND METHODS: Sixty patients with symptomatic atherosclerotic BA stenosis $\geq 70 \%$ were enrolled consecutively. HR-MRI of cross-sectional BAs was obtained before and after contrast media injection, and wall enhancement indices were calculated for sections proximal to, at, and distal to the site of maximal luminal narrowing. DWI of the brain was performed to determine the presence of recent infarction.

RESULTS: Images from 56 patients were suitable for analysis. Thirty-three patients underwent stent placement for the stenotic BA, and 23 patients underwent conservative medical treatment with antiplatelet agents and risk-factor control. All 23 patients with medical treatment had a 12-month follow-up. Greater wall enhancement was seen in the section proximal to the MLN section in both patients with recent infarction $(74 \pm 65 \%$ versus $44 \pm 44 \% ; P=.046)$ and in patients with subsequent ischemic events $(100 \pm 57 \%$ versus $44 \pm 44 \% ; P=$ .014).

CONCLUSIONS: Greater wall enhancement proximal to the MLN site correlates with recent infarction in the territory of the stenotic BA and subsequent ischemic events associated with the stenotic BA. Contrast-enhanced HR-MRI may serve as a noninvasive tool for risk stratification of BA atherosclerosis.

ABBREVIATIONS: $\quad$ BA = basilar artery; HR-MRI = high-resolution MRI; ICC = intraclass correlation coefficient; $\mathrm{MLN}=$ maximal luminal narrowing; $\mathrm{SI}=$ signal intensity

V lnerable plaques of coronary atherosclerosis are associated with an increased risk of acute coronary syndrome and sudden death. ${ }^{1}$ It has also been demonstrated that ischemic events or infarcts in the anterior cerebral circulation correlate well with

Received February 26, 2012; accepted after revision June 2

From the Department of Radiology (X.L., L.M.), People's Liberation Army General Hospital, Beijing, China; Department of Interventional Neuroradiology (N.M.), Tiantan Hospital, Capital Medical University, Beijing, China; and New Era Stroke Care and Research Institute (W.-J.J.), The Second Artillery General Hospital People's Liberation Army, Xicheng District, Beijing, China.

This work was supported by grants from the National Natural Science Foundation of China (81101034 to X. Lou; 81070925 to W.-J. Jiang) and by a grant from the Medical Capital Development Fund (2009-1937 to W.-J. Jiang). X. Lou and N. Ma contributed equally to this work.

Please address correspondence to W.-J. Jiang, MD, New Era Stroke Care and Research Institute, The Second Artillery General Hospital People's Liberation Army, No. 16 Xinwai St, Xicheng District, Beijing, 100088, China; e-mail: cjr.jiangweijian@vip.163.com

-- Indicates open access to non-subscribers at www.ajnr.org

http://dx.doi.org/10.3174/ajnr.A3241 atherosclerotic plaque vulnerability of the cervical carotid artery, as well as its degree of stenosis. ${ }^{2-4}$

A similar relation may exist between plaque vulnerability (such as the presence of calcification, intraplaque hemorrhage, surface disruption, or plaque enhancement) of intracranial arteries affected by atherosclerosis and subsequent ischemic events, including TIA and infarction in its arterial territory. ${ }^{5}$ Although there have been limited studies focusing on the issue, until recently no imaging technique has been available for in vivo human intracranial arterial plaque imaging.

HR-MRI has been increasingly used in recent years to study intracranial atherosclerotic plaque. ${ }^{6-10}$ Two previous case reports on intracranial atherosclerosis showed a link between recent infarction and qualitative assessment of wall enhancement in postcontrast HR-MRI. ${ }^{9,10}$ In this study, we used a quantitative method to assess the wall enhancement patterns of the BA in patients with symptomatic atherosclerotic BA stenosis at $3 \mathrm{~T}$ and to explore a relationship with recent infarction in the territory of 
the stenotic BA and with subsequent ischemic events associated with the stenotic BA.

\section{MATERIALS AND METHODS \\ Patients}

This study was approved by our institutional ethics committees, and written informed consent was obtained before each HR-MRI examination. From December 2007 to April 2012, 60 consecutive patients were enrolled in this study. The enrollment criteria were as follows: 1) ischemic stroke or transient ischemic attack in the posterior cerebral circulation within 90 days; 2 ) $\geq 70 \%$ BA stenosis verified by conventional angiography; and 3) 1 or more atherosclerotic risk factors, including hypertension, hyperlipidemia, diabetes mellitus, cigarette smoking, and obesity (body mass in$\operatorname{dex} \geq 30 \mathrm{~kg} / \mathrm{m}^{2}$ ). Using a traditional clinical definition of ischemic events, we defined ischemic stroke as a new focal neurologic deficit of sudden onset lasting $\geq 24$ hours and not caused by hemorrhage and TIA as acute onset of a focal neurologic deficit lasting $<24$ hours.

Patients with the following conditions were excluded: arteritis, contraindication to MRI, medical instability precluding MRI, concomitant bilateral vertebral artery stenoses of $\geq 50 \%$, no atherosclerotic risk factors, known atrial fibrillation on electrocardiogram (past or present), rheumatic heart disease, metallic heart valve, and/or myocardial infarction within 3 months. We screened a total of 64 patients, and 4 patients were excluded.

\section{MRI Protocol}

A 3T MR scanner (Signa, TwinSpeed; GE Healthcare, Milwaukee, Wisconsin) with an 8-channel phased-array head coil was used in this study. The gradient strength of the magnet was $23 \mathrm{mT} / \mathrm{m}$. DWI of the whole brain was performed in the axial plane with b-values of 0 and $1000 \mathrm{~s} / \mathrm{mm}^{2}$ by using a single-shot spin-echoplanar imaging sequence (TR/TE of $6500 / 110 \mathrm{~ms}, 128 \times 128 \mathrm{ma}-$ trix, $24 \times 24-\mathrm{cm}$ field of view, 1 excitation, and 5-mm thickness with a $0.5-\mathrm{mm}$ gap). Diffusion gradients were applied along 3 orthogonal directions ( $\mathrm{x}, \mathrm{y}$, and $\mathrm{z}$ axes).

The HR-MRI sequences consisted of 3D time-of-flight, T1WI, and postcontrast T1WI. Time-of-flight images were obtained by using $\mathrm{TR} / \mathrm{TE}=21 / 3.2 \mathrm{~ms}, \mathrm{FOV}=16 \times 16 \mathrm{~cm}$, thickness $=1 \mathrm{~mm}$, matrix $=256 \times 256$, and number of excitations $=1$. After localization of the MLN site on 3D time-of-flight MRA, a segment of 8 $\mathrm{mm}$ on T1WI was selected, which covered the site of MLN. If the 3D time-of-flight MRA showed that the BA course was severely tortuous, then the scan plane was angled to ensure that images were acquired perpendicular to the BA. T1WI was obtained by double inversion recovery fast spin-echo with the following parameters: TR/TI $/ \mathrm{TE}=800 / 650 / 8.6 \mathrm{~ms}, \mathrm{FOV}=16 \times 16 \mathrm{~cm}$, thickness $=2 \mathrm{~mm}$, matrix $=256 \times 320$, number of excitations $=4$, echo-train length $=12$. Postcontrast T1WI was acquired 5 minutes after gadolinium injection $(0.1 \mathrm{mmol} / \mathrm{kg}$ gadopentetate dimeglumine, Magnevist; Bayer Schering Pharma, Berlin, Germany) by using the same parameters as with the precontrast T1WI. In previous investigations, postcontrast enhancement was observed on black-blood T1WI in regions of fibrous carotid atherosclerotic plaque, suggesting that contrast-enhanced MRI acquired 5 minutes after the administration of Magnevist is valuable for assessing carotid plaque; thus, we used this method to explore the relation between wall enhancement and recent infarction in the territory of the stenotic BA. ${ }^{4}$ Fat suppression was used to reduce signal from surrounding fatty tissues in all sequences. A zip 512 matrix was used to enhance spatial resolution. The total imaging time of the $2 \mathrm{HR}$-MRI sequences used was 20 minutes.

\section{Identification of Recent Infarction}

DWI was evaluated by 1 reader (L.M.). Recent infarction was defined as an area of high signal intensity on DWI with a b-value of $1000 \mathrm{~s} /$ $\mathrm{mm}^{2}$. ADC maps were also reviewed to exclude the hyperintensity on DWI caused by T2 shinethrough effect. The infarction was further classified as within or outside of the territory of the stenotic BA as in a previous study. ${ }^{11}$

\section{Measurement of BA Wall Enhancement}

Matched precontrast and postcontrast T1WI of the BA was interpreted by 2 reviewers (X.L., N.M.) who reached a consensus opinion. The reviewers were blinded to clinical data. The images were graded on a 3 -point scale: $1=$ nonvisualization, 2 = adequate, and 3 good. Those with a score of 2 or 3 were analyzed after being zoomed to $400 \%$ by using FuncTool II software (GE Healthcare) from a GE workstation (Sun, ADW4.3). Three contiguous sections proximal to, at, and distal to the MLN site were selected to measure the extent of wall enhancement.

Wall enhancement index was used to characterize the extent of wall enhancement. After manually tracing the BA wall, SI of BA plaque $\left(\mathrm{SI}_{\text {plaque }}\right)$ at an unclosed-ring area on matched T1WI and postcontrast T1WI was measured. SI of adjacent normal gray matter $\left(\mathrm{SI}_{\text {gray matter }}\right)$, which was lesion-free in a round area of 18 $\mathrm{mm}^{2}$ on matched T1WI and postcontrast T1WI, was also measured as a reference (Figs 1 and 2). Then the wall enhancement index was calculated as follows: ( $\left[\mathrm{SI}_{\mathrm{plaque}} / \mathrm{SI}_{\text {gray matter }}\right.$ on postcontrast $\mathrm{T} 1 \mathrm{WI}]-\left[\mathrm{SI}_{\text {plaque }} / \mathrm{SI}_{\text {gray matter }}\right.$ on matched precontrast $\mathrm{T} 1 \mathrm{WI}]) /\left(\mathrm{SI}_{\text {plaque }} / \mathrm{SI}_{\text {gray matter }}\right.$ on matched precontrast $\left.\mathrm{T} 1 \mathrm{WI}\right) \times$ $100 \%$ SI $_{\text {plaque }}$ and $\mathrm{SI}_{\text {gray matter }}$ on matched precontrast and postcontrast T1WI in the initial 10 patients were measured by another pair of reviewers (N.M., L.M.) for estimation of interobserver variability.

\section{Occurrence of TIA and Stroke in Follow-Up}

At a 12-month follow-up, the ischemic events, including TIA and ischemic stroke associated with the stenotic BA in patients with medical treatment, were verified and recorded independently by a neurologist.

\section{Data Analysis}

Continuous variables were presented as mean \pm SD. Categoric variables were presented as percentages. Interobserver variability for the measurements of SI was determined with the use of ICC. Student $t$ test or Mann-Whitney $U$ test (when continuous variables had skewed distributions) was used to identify the difference in each of the variables between patients with and without recent infarction. Difference in each categoric variable between patients with and without recent infarction and patients with and without subsequent ischemic events was tested with the $\chi^{2}$ or Fisher exact 

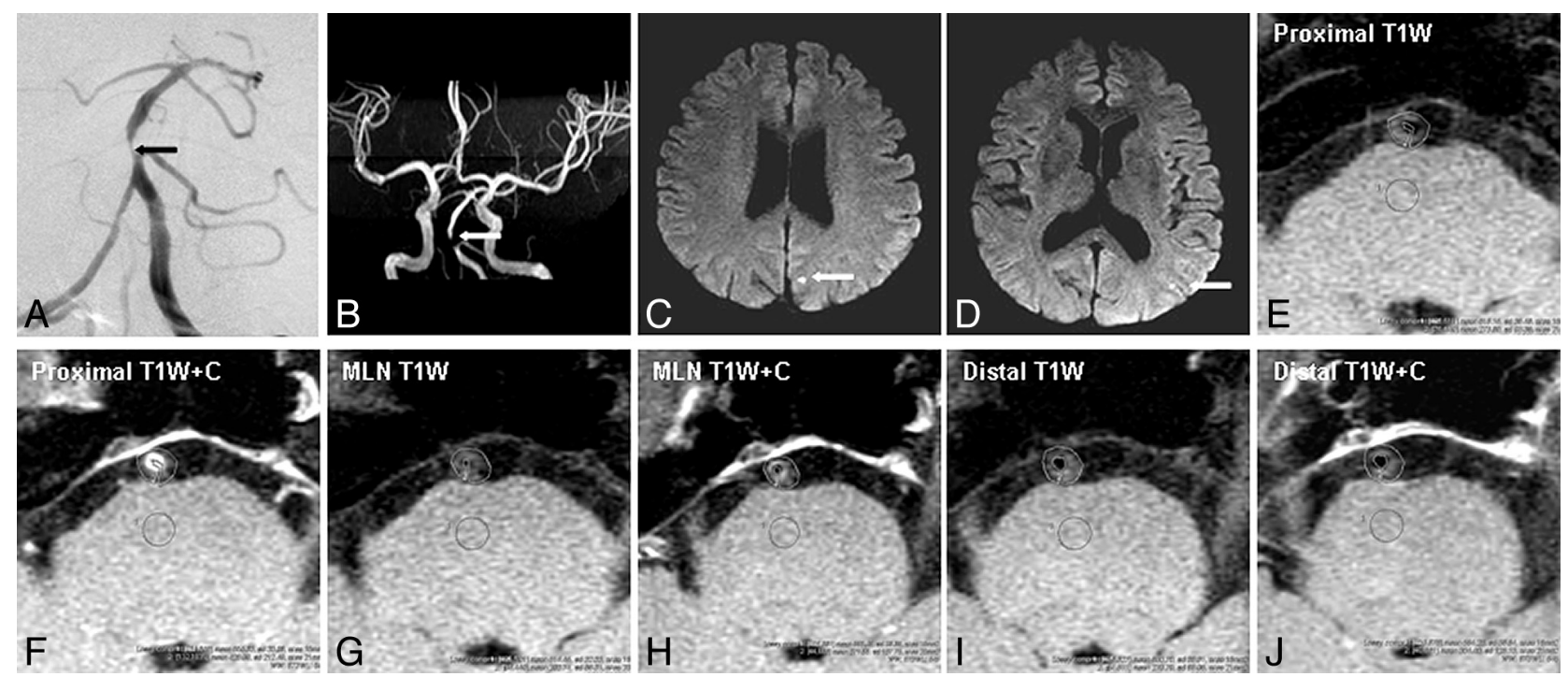

FIG 1. Images from a 58-year-old man with recurrent TIA symptoms of blurred vision, right limb numbness, slurred speech, and vertigo for 14 days. $A$ and $B, A$ severe stenosis at the proximal segment of the basilar artery is shown on conventional angiography (black arrow) and on MRA (white arrow). C and D, DWI demonstrates recent infarcts in the left occipital lobe that are in the territory of the stenotic basilar artery (white arrows). E-J, After matching TTWI and postcontrast TIWI at the section proximal to, at, and distal to the MLN site, an unclosed ring area is used for measuring signal intensity of the basilar artery wall and a round area of $18 \mathrm{~mm}^{2}$ for adjacent normal gray matter as a reference. The wall enhancement index at the section proximal to, at, and distal to the MLN site was $152 \%, 145 \%$, and $54 \%$, respectively. The strongest wall enhancement at the section proximal to the MLN site was observed.
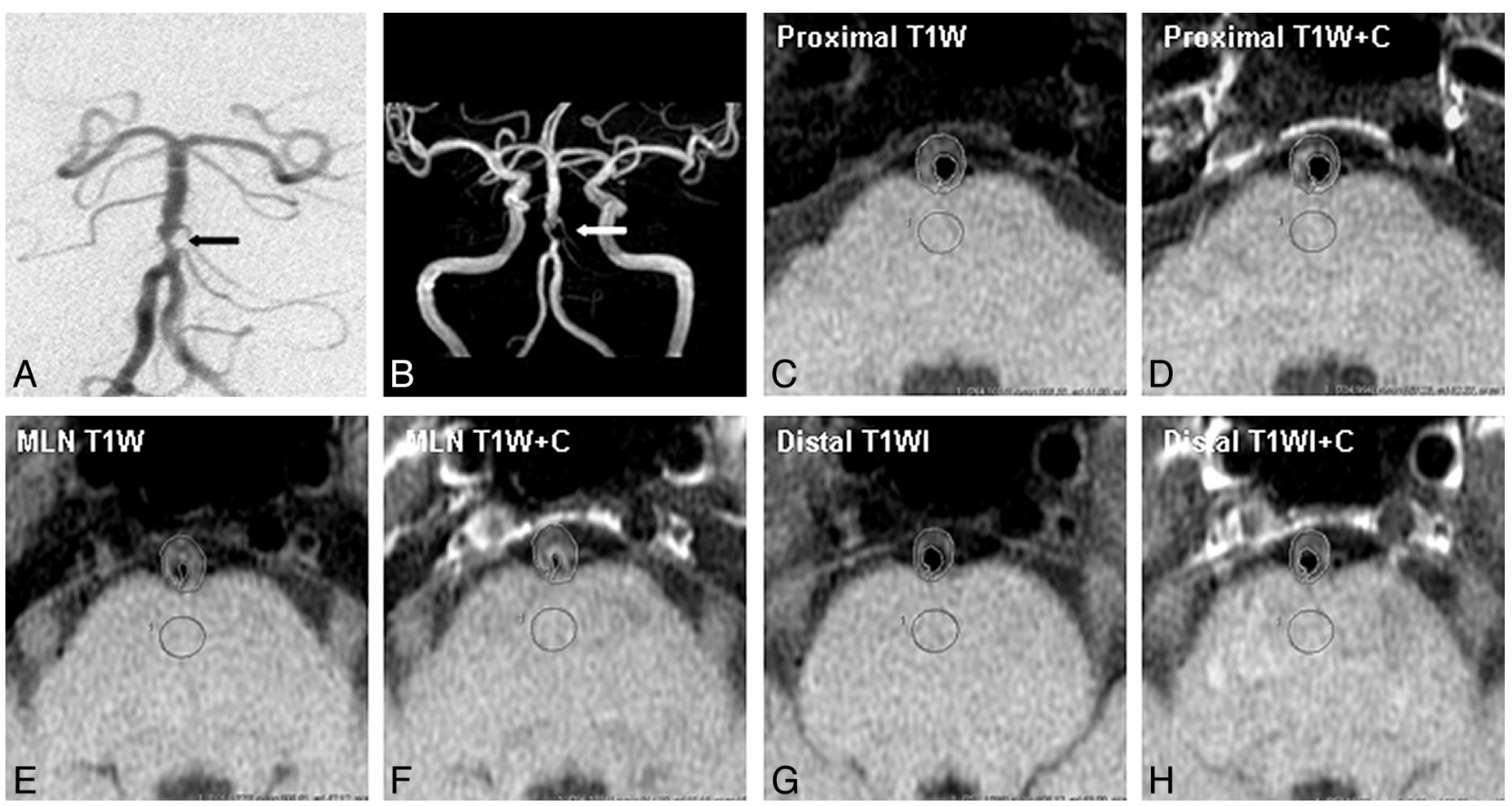

FIG 2. Images from a 66-year-old man with recurrent TIA symptoms of diplopia and left limb weakness for 22 days. $A$ and $B$, A severe stenosis at the proximal segment of basilar artery is shown on conventional angiography (black arrow) and on MRA (white arrow). DWI demonstrates no recent infarction in the territory of the stenotic basilar artery (not shown). $\mathrm{C}-\mathrm{H}$, After matching TIWI and postcontrast TIWI at the section proximal to, at, and distal to the MLN site, the wall enhancement index at the section proximal to, at, and distal to the MLN site was $14 \%, 29 \%$, and $22 \%$, respectively. The strongest wall enhancement was observed at the section of the MLN site.

test (when the expected cell frequency was $<5$ ). A value of 2 -tailed $P<.05$ was considered statistically significant.

\section{RESULTS}

Fifty-six patients, 36-75 years old, had images suitable for analysis (scores of 2 and 3). Among them, 50 patients were male, 47 pa- tients had hypertension, 51 patients had hyperlipidemia, 22 patients had diabetes mellitus, 34 patients had a history of cigarette smoking, and 7 patients were obese. The median time from the qualifying event to HR-MRI was 26 days (range, 1-88 days).

The stenotic lesion locations were the proximal segment of the $\mathrm{BA}(n=28[50 \%])$, the middle segment of the BA $(n=20[36 \%])$, 
Table 1: Characteristics in patients with and without recent infarction

\begin{tabular}{lccc}
\hline \multicolumn{1}{c}{ Characteristic } & $\begin{array}{c}\text { Infarction } \\
(\boldsymbol{n}=\mathbf{1 8})\end{array}$ & $\begin{array}{c}\text { Noninfarction } \\
(\boldsymbol{n}=\mathbf{3 8})\end{array}$ & $\boldsymbol{P}$ Value \\
\hline Age (years) & $57 \pm 8$ & $57 \pm 10$ & .872 \\
Male & $89 \%$ & $89 \%$ & 1.000 \\
Hypertension & $83 \%$ & $84 \%$ & 1.000 \\
Hyperlipidemia & $89 \%$ & $92 \%$ & .652 \\
Diabetes mellitus & $56 \%$ & $32 \%$ & .142 \\
Smoking & $67 \%$ & $58 \%$ & .573 \\
Obesity & $11 \%$ & $13 \%$ & 1.000 \\
Time from the qualifying & $29 \pm 26$ & $31 \pm 23$ & .823 \\
$\quad$ event to HRMRI (days) & & & \\
\hline
\end{tabular}

${ }^{\text {a }}$ Data are mean $\pm S D$.

Table 2: Comparison of wall enhancement index between patients with and without recent infarction

\begin{tabular}{lccc}
\hline \multicolumn{1}{c}{ Wall } & $\begin{array}{c}\text { Infarction } \\
\text { Enhancement Index }\end{array}$ & $\begin{array}{c}\text { Noninfarction } \\
(\boldsymbol{n}=\mathbf{1 8})\end{array}$ & $\boldsymbol{P}$ Value \\
\hline At the proximal section & $74 \pm 65 \%$ & $44 \pm 44 \%$ & .046 \\
At the MLN site & $69 \pm 41 \%$ & $54 \pm 42 \%$ & .229 \\
At the distal section & $42 \pm 37 \%$ & $40 \pm 39 \%$ & .827 \\
\hline
\end{tabular}

Note:-Unless otherwise indicated, data are mean \pm SD.

and the boundary of BA and intracranial vertebral artery $(n=8$ [14\%]). The bilateral vertebral arteries were equal in size in 24 patients $(43 \%)$ and unequal in 32 patients $(57 \%)$. One of the bilateral vertebral arteries was hypoplastic or absent in 16 patients (29\%). Asymptomatic anterior circulation stenosis was observed in 9 patients $(16 \%)$, mild $(<50 \%)$ vertebral artery stenosis in 5 ( $9 \%$ ), severe ( $\geq 70 \%$ ) ostial stenosis on the side of the hypoplastic vertebral artery with ipsilateral intracranial segment absence in 4 $(7 \%)$, mild $(<50 \%)$ posterior cerebral artery stenosis in $3(5 \%)$, and a unilateral posterior cerebral artery in $4(7 \%)$.

The interobserver reproducibility was excellent for measurements of $\mathrm{SI}_{\text {plaque }}(\mathrm{ICC}=0.893 ; 95 \% \mathrm{CI}: 0.827-0.935)$ and $\mathrm{SI}_{\text {gray matter }}(\mathrm{ICC}=0.939 ; 95 \% \mathrm{CI}: 0.900-0.963)$ on T1WI, and $\mathrm{SI}_{\text {plaque }}(\mathrm{ICC}=0.975 ; 95 \% \mathrm{CI}: 0.959-0.985)$ and $\mathrm{SI}_{\text {gray matter }}$ (ICC $=0.958 ; 95 \%$ CI: 0.931-0.975) on postcontrast T1WI.

Wall enhancement indices for the BA proximal to, at, and distal to the MLN site were $54 \pm 53 \%, 58 \pm 42 \%$, and $41 \pm 38 \%$, respectively. Wall enhancement index of the section distal to the MLN site was lower than both the proximal section $(P=.048)$ and the section at the MLN site $(P=.001)$. There was no significant difference in the wall enhancement index between the proximal section and the section with $\operatorname{MLN}(P=0.566)$.

DWI showed that 22 patients $(39 \%)$ had recent infarction, including 18 patients (32\%) with infarction in the territory of the stenotic BA. There was no difference in patient characteristics between the 18 patients with infarction in the territory of the stenotic BA and the 38 patients without infarction in the stenotic BA distribution (Table 1).

Compared with patients without recent infarction in the distribution of the stenotic BA, those with infarction had a greater wall enhancement index on the section proximal to the MLN site (74 $\pm 65 \%$ versus $44 \pm 44 \%$, respectively; $P=.046)$. There was no significant difference in wall enhancement index between the section containing the MLN site and the section distal to the MLN site (Table 2).

Thirty-three patients underwent stent placement for the stenotic BA, and 23 patients underwent conservative medical treat-
Table 3: Comparison of wall enhancement index between

patients with and without subsequent ischemic events

\begin{tabular}{lccc} 
Wall & $\begin{array}{c}\text { Subsequent } \\
\text { Ischemic Events } \\
\text { Enhancement Index }\end{array}$ & $\begin{array}{c}\text { No Subsequent } \\
\text { Ischemic Events }\end{array}$ & \\
\hline At the proximal section & $100 \pm 57 \%$ & $44 \pm 44 \%$ & .014 \\
At the MLN site & $82 \pm 39 \%$ & $55 \pm 39 \%$ & .113 \\
At the distal section & $46 \pm 47 \%$ & $32 \pm 43 \%$ & .489 \\
\hline
\end{tabular}

Note:- Unless otherwise indicated, data are mean \pm SD

ment with antiplatelet agents and risk-factor control. All 23 patients with medical treatment had a 12-month follow-up. Recurrence of ischemic events in these patients was found in 9 patients, including 7 with TIA and 2 with stroke. Compared with patients without the subsequent ischemic events, those with the subsequent ischemic events had a greater wall enhancement index at the section proximal to the MLN site (100 $\pm 57 \%$ versus $44 \pm$ $44 \%$, respectively; $P=.014$ ). No significant difference in the wall enhancement index in the section at and distal to the MLN site was seen (Table 3).

\section{DISCUSSION}

Enhancement of the wall of an intracranial artery can occasionally be seen on routine MR imaging, as well as on HR-MRI in elderly patients, but an enhancement evaluation of the intracranial arterial wall by using a quantitative method has not been performed. ${ }^{9,10}$ With contrast enhancement, arterial wall thickening was more clearly and easily observed on HR-MRI. The BA is the largest artery in the posterior circulation and forms the central core of this vascular territory. It is commonly affected by atherosclerosis, and previous studies have found it to be among the first and most severely involved of all intracranial arteries. ${ }^{5}$ We therefore performed this prospective study on the BA because it is a preferred site for initial evaluation of intracranial arterial wall enhancement. Wall enhancement in patients with symptomatic atherosclerotic BA stenosis can be calculated on HR-MRI with the techniques outlined in this study. Contrast enhancement suggests the presence of a vascular supply to the plaque and thus increased endothelial permeability that facilitates the entry of the contrast agent from the blood plasma. ${ }^{12,13}$ Histologic studies have verified that carotid plaque enhancement on postcontrast T1WI is related to extensive neovasculaturization and accumulation of macrophages and T cells, reflecting plaque vulnerability. ${ }^{14,15}$

This study demonstrated that wall enhancement is not equally distributed along the BA. A higher wall enhancement index was observed at the section proximal to or at the MLN site than distal to the MLN site in all patients. The current study shows that wall plaque enhancement of the proximal plaque was associated not only with recent infarction but also with subsequent ischemic stroke, which may suggest that the proximal plaque with obvious enhancement is more vulnerable. A similar situation is also found in the studies focusing on coronary and extracranial carotid arteries. In a study on coronary atherosclerosis, plaque rupture was more frequently observed at the proximal, upstream side of the site of maximal stenosis, which is exposed to higher wall shear stress. ${ }^{16-18}$ In patients with symptomatic extracranial carotid atherosclerotic plaques, the upstream side of the stenosis had higher incidence of cap rupture and intraplaque hemorrhage with more 
macrophages, fewer smooth muscle cells, and more collagen compared with the downstream side. ${ }^{18}$

This study had some limitations. First, the sample size was small. Second, the findings by HR-MRI have no histologic verification because of the difficulty of obtaining specimens of the BA for histology. Third, because T1WI has a long scanning time, only an 8-mm section of the BA was covered with T1WI, and this small section may not be representative of the entire plaque. Finally, this study focused only on symptomatic patients, so a prospective study is needed of patients with symptomatic and asymptomatic BA stenosis to further understand the pathogenesis and natural history of BA atherosclerosis.

\section{CONCLUSIONS}

Our study demonstrated that wall enhancement is not equally distributed along the course of the atherosclerosis-involved BA and that proximal enhancement is associated with a higher rate of both recent infarction and subsequent ischemic stroke.

\section{ACKNOWLEDGMENTS}

We thank Dr. Lin-Feng Zhang, MD, PhD, at Department of Epidemiology, Fuwai Hospital of the Chinese Academy of Medical Sciences and Peking Union Medical College, Beijing, China, who assisted with statistical analysis; Hao Shen, $\mathrm{PhD}$, at Applied Science Laboratory, GE Healthcare, for his help in improvement of MR imaging protocol; and Ke-Hui Dong, MD, at the Department of Neurology, Tiantan Hospital, Capital Medical University, Beijing, China, for her help in follow-up of patients in this study.

\section{REFERENCES}

1. Kusama I, Hibi K, Kosuge M, et al. Impact of plaque rupture on infarct size in ST-segment elevation anterior acute myocardial infarction. J Am Coll Cardiol 2007;50:1230-37

2. Zhao X, Zhao Q, Chu B, et al. Prevalence of compositional features in subclinical carotid atherosclerosis determined by high-resolution magnetic resonance imaging in Chinese patients with coronary artery disease. Stroke 2010;41:1157-62

3. Saam T, Hatsukami TS, Takaya N, et al. The vulnerable, or high-risk, atherosclerotic plaque: noninvasive MR imaging for characterization and assessment. Radiology 2007;244:64-77

4. Ouhlous M, Flach HZ, de Weert TT, et al. Carotid plaque composi- tion and cerebral infarction: MR imaging study. AJNR Am J Neuroradiol 2005;26:1044-49

5. Voetsch B, DeWitt LD, Pessin MS, et al. Basilar artery occlusive disease in the New England Medical Center Posterior Circulation Registry. Arch Neurol 2004;61:496-504

6. Klein IF, Lavallée PC, Mazighi M, et al. Basilar artery atherosclerotic plaques in paramedian and lacunar pontine infarctions: a high-resolution MRI study. Stroke 2010;41:1405-09

7. Ma N, Jiang WJ, Lou X, et al. Arterial remodeling of advanced basilar atherosclerosis: a 3-Tesla MRI study. Neurology 2010;75:253-58

8. Ma N, Lou X, Zhao TQ, et al. Intraobserver and interobserver variability for measuring the wall area of the basilar artery at the level of the trigeminal ganglion on high-resolution MR images. AJNR Am J Neuroradiol 2011;32:e29-32

9. Vergouwen MD, Silver FL, Mandell DM, et al. Eccentric narrowing and enhancement of symptomatic middle cerebral artery stenoses in patients with recent ischemic stroke. Arch Neurol 2011;68:338-42

10. Aoki S, Shirouzu I, Sasaki Y, et al. Enhancement of the intracranial arterial wall at MR imaging: relationship to cerebral atherosclerosis. Radiology 1995;194:477-81

11. Tatu L, Moulin T, Bogousslavsky J, et al. Arterial territories of human brain: brainstem and cerebellum. Neurology 1996;47:1125-35

12. Yuan C, Kerwin WS, Ferguson MS, et al. Contrast-enhanced high resolution MRI for atherosclerotic carotid artery tissue characterization. J Magn Reson Imaging 2002;15:62-67

13. Cai J, Hatsukami TS, Ferguson MS, et al. In vivo quantitative measurement of intact fibrous cap and lipid-rich necrotic core size in atherosclerotic carotid plaque: comparison of high-resolution, contrast-enhanced magnetic resonance imaging and histology. Circulation 2005;112:3437-44

14. Mofidi R, Crotty TB, McCarthy P, et al. Association between plaque instability, angiogenesis and symptomatic carotid occlusive disease. Br J Surg 2001;88:945-50

15. McCarthy MJ, Loftus IM, Thompson MM, et al. Angiogenesis and the atherosclerotic carotid plaque: an association between symptomatology and plaque morphology. J Vasc Surg 1999;30:261-68

16. Box LC, Angiolillo DJ, Suzuki N, et al. Heterogeneity of atherosclerotic plaque characteristics in human coronary artery disease: a three-dimensional intravascular ultrasound study. Catheter Cardiovasc Interv 2007;70:349-56

17. Lovett JK, Rothwell PM. Site of carotid plaque ulceration in relation to direction of blood flow: an angiographic and pathological study. Cerebrovasc Dis 2003;16:369-75

18. Fagerberg B, Ryndel M, Kjelldahl J, et al. Differences in lesion severity and cellular composition between in vivo assessed upstream and downstream sides of human symptomatic carotid atherosclerotic plaques. J Vasc Res 2010;47:221-30 